\title{
Éclosion d'infection à Shigella sonnei dans la communauté juive ultra-orthodoxe de Montréal, 2015
}

\author{
Pilon $\mathrm{PA}^{1,2^{\star}}$, Camara B ${ }^{1}$, Bekal $\mathrm{S}^{3,4}$
}

\section{Résumé}

Une éclosion d'infection à Shigella sonnei survenue dans la communauté juive ultra-orthodoxe (CJUO) a fait l'objet d'une investigation et d'une intervention par la Direction régionale de santé publique de Montréal (DRSP) en collaboration avec plusieurs partenaires de la santé et du milieu communautaire. Un total de 27 cas confirmés a été rapporté dans cette éclosion entre les mois de février et de juin 2015. La courbe épidémique est compatible avec une source ponctuelle avec transmission secondaire de personne à personne. L'analyse des souches par électrophorèse sur gel en champ pulsé pour 11 des 27 cas a mis en évidence un seul profil électrophorétique nouvellement identifié au Québec. La presque totalité des souches analysées montraient une résistance à l'ampicilline et au triméthoprime-sulfaméthoxazole. Les cas habitaient tous dans le Centre-Ouest de Montréal. La majorité des cas étaient âgés de moins de cinq ans et fréquentaient un milieu de garde soit un milieu reconnu propice à la transmission de maladies entériques. Les communications acheminées précocement aux familles, aux différents intervenants des milieux de garde et scolaires, aux partenaires communautaires et aux synagogues de la CJUO par la DRSP ont pu contribuer à réduire la transmission de la shigellose dans cette communauté.

\section{Affiliations}

'Secteur Prévention et contrôle des maladies infectieuses, Direction régionale de santé publique de Montréal, Montréal (Québec)

2École de santé publique, Université de Montréal, Montréal (Québec)

${ }^{3}$ Laboratoire de santé publique du Québec, Sainte-Anne-de-Bellevue (Québec)

${ }^{4}$ Département de microbiologie, d'infectiologie et d'immunologie, Université de Montréal, Montréal (Québec)

*Correspondance : ppilon@ santepub-mtl.qc.ca

Citation proposée: Pilon PA, Camara B, Bekal S. Éclosion d'infection à Shigella sonnei dans la communauté juive ultra-orthodoxe de Montréal, 2015. Relevé des maladies transmissibles au Canada 2016;42:98-102. https://doi.org/10.14745/ccdr.v42i04a03f

\section{Contexte}

Le 25 mars 2015, la Direction régionale de santé publique de Montréal (DRSP) détectait par analyse SaTScan ${ }^{\mathrm{MC}}$ un agrégat spatio-temporel statistiquement significatif de 7 cas de shigellose déclarés dans les 12 jours précédents (du 13 au 25 mars). Les premières enquêtes épidémiologiques révélaient que 3 des 7 cas étaient survenus chez des enfants de la communauté juive ultra-orthodoxe (CJUO). Les quatre autres cas avaient contracté leur infection en voyage et n'avaient aucun lien entre eux. Cet agrégat avait été précédé par la déclaration le 25 février d'un cas chez un enfant fréquentant un milieu de garde de cette communauté dont les symptômes ont débuté le 19 février. Sur la base des données épidémiologiques et historiques, une éclosion de shigellose dans la CJUO montréalaise a donc été fortement suspectée et une investigation a été initiée.

Parallèlement, une éclosion comportant 43 cas d'infection à $S$. sonnei touchant deux communautés similaires faisait l'objet d'une alerte de santé publique de la ville de New York en décembre 2014 (1). En raison des voyages réguliers des membres de la CJUO entre Montréal et New York, il était important d'investiguer un possible lien entre les deux éclosions. Les objectifs de cette investigation étaient de mieux caractériser I'éclosion de S. sonnei dans la CJUO, d'élaborer des hypothèses et de guider les interventions éventuelles de la DRSP. Un rapport d'investigation a été rédigé afin de partager les stratégies d'intervention utilisées et pour servir de document de référence pour des investigations ultérieures portant sur le même sujet.

\section{Méthodologie}

\section{Définition de cas}

Un cas a été défini comme étant un résident de Montréal ayant une confirmation par laboratoire d'une infection à $S$.

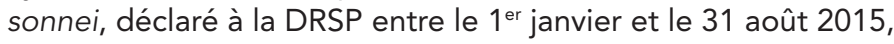
appartenant à la CJUO et n'ayant pas d'histoire de voyage récent à l'étranger.

\section{Recherche des cas et collecte des données}

La recherche des cas a été réalisée au moyen de la base de données des maladies à déclaration obligatoire (MADO). La collecte des données a été effectuée, d'une part, par l'exploitation du fichier MADO et, d'autre part, par l'examen des dossiers d'enquête épidémiologique des cas. L'attribution d'un cas à la CJUO ou d'un groupe d'appartenance (ex. : Belz, Satmar, etc.) a été basée sur les réponses obtenues lors de l'enquête. 


\section{Analyses de laboratoire}

Les analyses de laboratoire ont été effectuées dans les laboratoires de microbiologie médicale des hôpitaux déclarants (identification du genre, de l'espèce et du profil de sensibilité). La confirmation de l'identification ainsi que l'électrophorèse sur gel en champs pulsés (EGCP) a été effectuée au Laboratoire de santé publique du Québec (LSPQ).

\section{Analyse épidémiologique}

Une liste des cas a été générée et importée dans le logiciel Microsoft Excel 2010; cette liste incluait des variables démographiques, cliniques et épidémiologiques. Les analyses descriptives ont été effectuées avec le logiciel SPSS version 12.0.2.

\section{Intervention de santé publique}

Chaque cas déclaré a fait l'objet d'une enquête auprès de la famille. Des renseignements sur la prévention ont été donnés à la famille du cas et au milieu qu'il fréquente (milieux de garde ou scolaires). Compte tenu du contexte d'éclosion, des démarches ont également été effectuées auprès de deux partenaires communautaires de la CJUO afin d'informer leur population par l'intermédiaire des synagogues et des milieux de garde et scolaires, et de renforcer les mesures d'hygiène.

Les partenaires du réseau de la santé ont été informés ainsi que le LSPQ pour augmenter la vigilance des professionnels de la santé (surveillance rehaussée) et obtenir les analyses de laboratoire de confirmation et de caractérisation.

\section{Résultats}

\section{Description des cas en fonction du temps}

Entre le 19 février et le 1 ${ }^{\text {er }}$ juin 2015, 27 cas confirmés d'infection à $S$. sonnei (contractée localement) sont survenus chez des résidents montréalais appartenant à la CJUO. Cela représentait $79 \%(27 / 34)$ de l'ensemble des cas confirmés d'infection à $S$. sonnei déclarés dans la région pour la même période. Le premier cas a été observé le 19 février et l'éclosion a persisté cinq mois. Le sommet est survenu durant le mois de mai avec 10 cas recensés soit environ $37 \%$ des cas. Ensuite, il y a eu une

Figure 1 : Courbe épidémique de l'éclosion de Shigella sonnei dans la communauté juive ultra-orthodoxe, région de Montréal, de février à juin $2015^{1}$

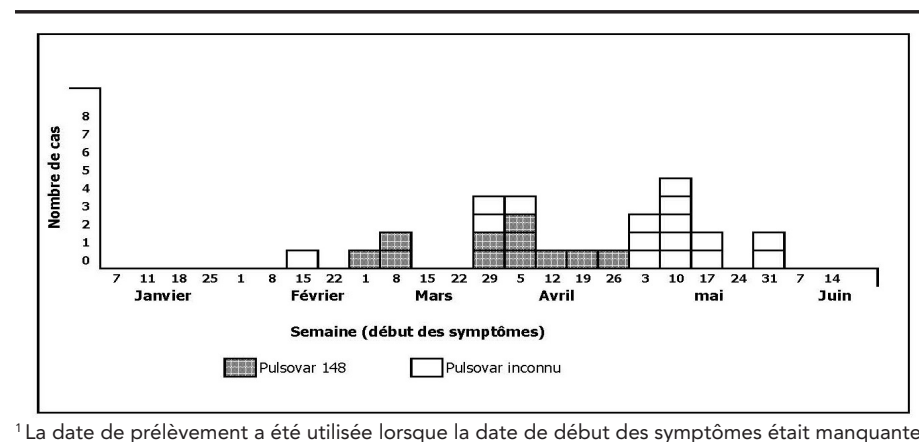
$(n=4$ cas $)$ diminution au mois de juin et aucun cas n'a été observé durant les mois de juillet et d'août. En tenant compte de la période d'incubation minimale et maximale de 1 et 3 jours, la période d'exposition des cas se situait entre le 18 février et le 28 mai 2015. La courbe épidémique (figure 1) est en faveur d'une source ponctuelle avec une transmission secondaire de personne à personne.

\section{Description des cas en fonction du milieu}

Tous les cas résidaient dans le Centre-Ouest de Montréal. Onze sur 27 cas partageaient le même code postal. Pour 23 des 27 cas, les milieux fréquentés durant leur période de contagiosité ont été précisés. II s'agissait soit d'un milieu de garde (8 au total) pour 13 cas (57\%), d'une école primaire (3 au total) pour 5 cas (22\%), du domicile pour 4 cas (17\%) et d'une université pour 1 cas (4\%). Un milieu de garde et une école se sont démarqués avec 3 cas associés chacun.

\section{Description des caractéristiques des cas}

L'âge variait de 1 à 35 ans avec une moyenne de 10 ans et une médiane de 4 ans. On comptait 15 (56\%) hommes et 12 (44 \%) femmes pour un ratio H/F de 1,3. La majorité (74 \%) des cas étaient des enfants âgés de moins de 10 ans (52\% âgés de moins de 5 ans) [figure 2]. II est intéressant de noter que les cas rapportés chez les adultes touchent exclusivement des femmes; ce qui est probablement dû au fait que les femmes s'occupent davantage des soins aux enfants.

Figure 2 : Nombre de cas d'infection à Shigella sonnei dans la communauté juive ultra-orthodoxe, par groupe d'âge et par sexe, région de Montréal, de février à juin 2015

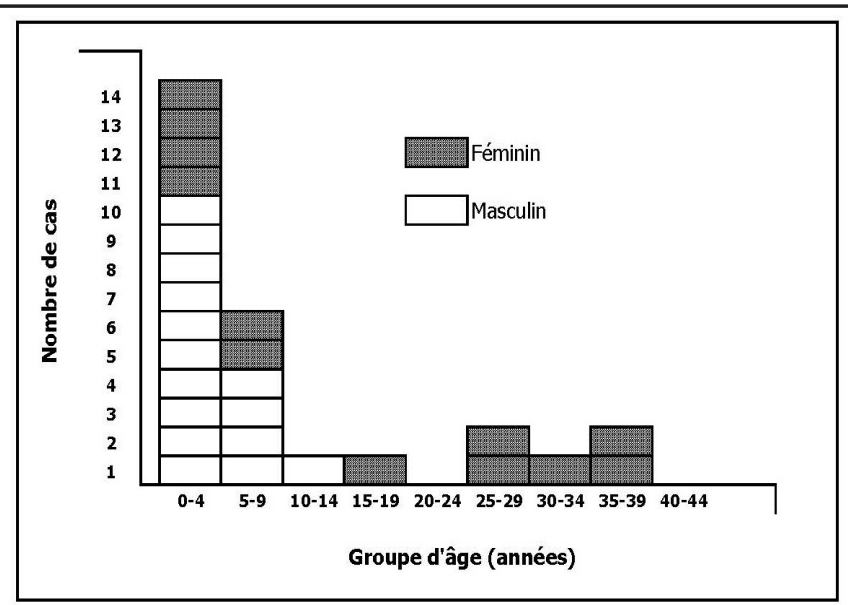

\section{Présentation clinique}

L'information sur les signes et symptômes a été obtenue pour 22 des 27 cas (tableau 1). La fièvre accompagnée de symptômes digestifs bas ont été les symptômes les plus fréquents. La présence de fièvre et de sang dans les selles témoignent d'une maladie plus grave chez plus de la moitié des cas (55\%) [tableau 1]. 
Tableau 1 : Fréquence des symptômes des cas d'infection à Shigella sonnei en fonction des groupes d'âge ( $\mathrm{N}=22)$

\begin{tabular}{|l|r|r|r|}
\hline \hline Tableau clinique & $\begin{array}{r}0 \text { à } 9 \text { ans } \\
N=15(\%)\end{array}$ & $\begin{array}{r}10 \text { à } 39 \text { ans } \\
N=7(\%)\end{array}$ & $\begin{array}{c}\text { Tous les âges } \\
N=22(\%)\end{array}$ \\
\hline Diarrhée & $15(100)$ & $7(100)$ & $22(100)$ \\
\hline $\begin{array}{l}\text { Crampes/douleurs } \\
\text { abdominales }\end{array}$ & $14(93)$ & $5(71)$ & $19(86)$ \\
\hline Fièvre $\left(\geq 38^{\circ} \mathrm{C}\right)$ & $13(86)$ & $5(71)$ & $18(82)$ \\
\hline Sang dans les selles & $10(67)$ & $3(43)$ & $13(59)$ \\
\hline Fatigue inhabituelle & $9(60)$ & $3(43)$ & $12(55)$ \\
\hline Nausées & $7(47)$ & $4(57)$ & $11(50)$ \\
\hline Vomissements & $8(53)$ & $1(14)$ & $9(41)$ \\
\hline
\end{tabular}

Abréviation: $\mathrm{N}$, nombre de cas

\section{Consultation médicale et hospitalisation}

Le premier contact avec le système de soins a eu lieu dans une clinique ambulatoire pour 24 cas (89\%) et à l'urgence d'un hôpital pour 3 cas (11\%). Parmi les 24 cas ayant consulté dans une clinique, 18 (75\%) ont consulté à une même clinique située sur leur territoire de résidence et qui semble desservir la CJUO.

Aucun des 27 cas n'a été hospitalisé ou n'est décédé au moment de l'enquête.

\section{Traitement}

Parmi les 25 cas ayant fourni l'information sur le traitement, 17 $(68 \%)$ ont reçu un traitement antibiotique dont $9(53 \%)$ de la ciprofloxacine. Un cas a reçu de l'ampicilline malgré le profil de résistance de la souche en cause (tableau 2 ).

\section{Résultats de laboratoire}

Tous les cas ont été confirmés en laboratoire par coproculture. Un résultat d'antibiogramme était disponible pour 24 des 27 cas et tous avaient une résistance à l'ampicilline ou au sulfaméthoxazole-triméthoprime (SMX/TMP) [tableau 3]. Pour tous les cas sauf deux (1 ciprofloxacine et 1 céfixime), nous n'avons aucune donnée sur la sensibilité à ces deux antibiotiques ainsi que sur l'azithromycine. L'EGCP a été effectuée pour

Tableau 2 : Traitement antibiotique des cas d'infection à Shigella sonnei $(\mathrm{N}=25)$

\begin{tabular}{|l|r|}
\hline \hline \multicolumn{1}{|c|}{ Traitement antibiotique } & Nombre (\%) \\
\hline Ciprofloxacine & $9(53)$ \\
\hline Azithromycine & $2(12)$ \\
\hline Cefixime & $2(12)$ \\
\hline Cephalexine & $1(6)$ \\
\hline Ampicilline & $1(6)$ \\
\hline Inconnu & $2(12)$ \\
\hline Total & $17(100)$ \\
\hline
\end{tabular}

'Le total est de plus de $100 \%$ en raison de l'arrondissement des nombres
Tableau 3 : Profil de résistance des souches de Shigella sonnei selon l'antibiogramme $(\mathrm{N}=24)$

\begin{tabular}{|l|r|}
\hline \multicolumn{1}{|c|}{ Profil de résistance } & Nombre (\%) \\
\hline Ampicilline (R) + TMP/SMX (R) & $6(67)$ \\
\hline Ampicilline (R) + TMP/SMX (I) & $1(4)$ \\
\hline Ampicilline (R) & $1(4)$ \\
\hline TMP/SMX (I) & $24(100)$ \\
\hline Total &
\end{tabular}

11 des 27 cas et un seul profil génétique, le pulsovar 148, a été mis en évidence. Ce pulsovar n'avait pas été identifié au Québec dans le passé et est distinct du profil électrophorétique de la souche ayant causé une éclosion dans la région de New York en décembre 2014.

\section{Sources potentielles d'exposition}

Parmi les 27 cas, 5 avaient un lien familial avec un autre cas confirmé déjà déclaré à la DRSP. Huit sur 27 cas ont indiqué avoir eu un contact avec un cas présentant une diarrhée avant le début de leur maladie (incluant 3 contacts de cas confirmés). II s'agissait d'un membre de la famille pour 4 cas et un contact seulement avec un milieu de garde ou une école primaire pour les 4 autres cas. Le cas index de cette éclosion était un garçon de 2 ans dont les symptômes ont débuté en février et qui fréquentait un milieu de garde (nom non précisé). Trois membres de sa famille avaient également eu de la diarrhée (séquence temporelle inconnue). Le cas et sa famille n'avaient pas effectué de voyage récent. Les cinq cas qui ont suivis au mois de mars (dont 4 ayant un pulsovar identique) étaient également des enfants âgés entre 4 et 10 ans qui fréquentaient des écoles primaires ou des milieux de garde différents et qui ne semblaient pas avoir de lien évident avec le cas index. Toutefois, ces cas appartenaient à un même groupe de la CJUO et auraient pu participer à une activité commune donnant lieu à une transmission interhumaine. En ce qui concerne les cas survenus en avril et en juin, certains cas étaient des fratries de cas précédents survenus probablement par transmission intrafamiliale. Une transmission a pu également survenir dans deux milieux de garde (centre de la petite enfance [CPE] A et $B$ ) et dans une école primaire (école primaire A) [cas initial suivi d'autres cas rapprochés dans le temps] (tableau 4).

\section{Intervention de santé publique}

Dans la présente investigation, chaque cas déclaré de shigellose confirmé par le laboratoire a fait l'objet d'une intervention. Cette dernière portait sur la nécessité d'attendre au moins 48 heures après la fin de la diarrhée avant de réintégrer le milieu de garde ou le milieu scolaire. De plus, un feuillet d'information sur la prévention de la shigellose a été acheminé aux parents d'un enfant affecté et au milieu fréquenté de façon à augmenter la vigilance des responsables des différents milieux et des autres parents et à renforcer les mesures préventives. 
Tableau 4 : Distribution des cas confirmés d'infection à Shigella sonnei selon le milieu fréquenté $(N=27)$

\begin{tabular}{|l|l|r|}
\hline \multicolumn{1}{|c|}{ Milieu fréquenté } & \multicolumn{1}{|c|}{ Nom du milieu } & Nombre de cas \\
\hline \multirow{4}{*}{$\begin{array}{l}\text { Milieu de garde }(\mathrm{N}=13) \\
\end{array}$} & CPE A & 3 \\
\cline { 2 - 3 } & CPE B & 2 \\
\cline { 2 - 3 } & CPE C (filles) & 1 \\
\cline { 2 - 3 } & CPE D (garçons) & 1 \\
\cline { 2 - 3 } & CPE E & 1 \\
\cline { 2 - 3 } & CPE F & 1 \\
\cline { 2 - 3 } & Garderie G (filles) & 1 \\
\cline { 2 - 3 } & Garderie H & 1 \\
\cline { 2 - 3 } & Inconnu & 2 \\
\hline \multirow{5}{*}{ École primaire $(\mathrm{N}=5)$} & A (garçons) & 3 \\
\cline { 2 - 3 } & B (filles) & 1 \\
\cline { 2 - 3 } & C (filles) & 1 \\
\hline Université $(\mathrm{N}=1)$ & A & 4 \\
\hline Autres $(\mathrm{N}=8)$ & Domicile & 4 \\
\hline & Inconnu & 1 \\
\hline
\end{tabular}

Abréviations : $\mathrm{CPE}$, centre de la petite enfance; $\mathrm{N}$, nombre de cas

\section{Discussion}

Les éclosions de shigellose sont cycliques dans la communauté juive ultra-orthodoxe (CJUO). À Montréal, des éclosions d'infection à $S$. sonnei d'intensité variable sont survenues à des intervalles d'environ 1 à 5 ans (tableau 5) [2-9]. La réapparition régulière de la shigellose dans la CJUO serait le résultat d'introductions de l'agent infectieux liées aux voyages dans d'autres communautés similaires ayant une prévalence élevée de la maladie ou par la présence de porteurs chroniques qui serviraient de réservoir $(2,5,10)$. Quant à la périodicité des éclosions de Shigella dans la CJUO, elle pourrait s'expliquer par une faible endémicité persistante qui engendre une éclosion une fois qu'une nouvelle cohorte de jeunes enfants sans shigellose antérieure entre en milieux de garde ou scolaires (3).

La propagation de la shigellose dans cette éclosion résulte d'une transmission intrafamiliale (4 des 27 cas confirmés sont des fratries et plusieurs autres cas ont eu un contact avec des membres de famille souffrant de diarrhée) et d'une transmission en milieux de garde ( 13 des 27 cas) et scolaire ( 5 des 27 cas sont liés à une école primaire). Des contacts étroits, la fréquentation d'un milieu de garde, le fait d'avoir plusieurs jeunes enfants à la maison sont d'ailleurs considérés comme des facteurs de risque dans les éclosions déjà publiées $(3,5)$. L'agrégation spatiotemporelle des cas et la diversité des milieux laissent penser à une transmission de personne à personne. L'apparition de cas dans plusieurs groupes de la CJUO est un argument en faveur du rôle des milieux communautaires (en plus du milieu familial) dans la transmission. Les caractéristiques de l'éclosion montréalaise sont comparables à celles décrites dans d'autres villes (2-5). Les habitudes d'hygiène non développées chez les jeunes enfants et une dose infectieuse faible pour la transmission de $S$. sonnei diminuent l'efficacité des mesures préventives dans cette population (3).
Tableau 5 : Historique des éclosions d'infection à Shigella sonnei dans la communauté juive ultra-orthodoxe de la région de Montréal, de 1994 à 2015

\begin{tabular}{|l|l|}
\hline \multicolumn{1}{|c|}{ Période } & \multicolumn{1}{c|}{ Nombre de cas confirmés } \\
\hline Février et juin 2015 & 27 (pulsovar 148) \\
\hline Août 2011 à décembre 2012 (8) & 38 (plusieurs pulsovars) \\
\hline Novembre 2007 à janvier $2008(7)$ & 11 (pulsovar 35 et apparentés) \\
\hline Octobre 2004 à juillet 2005 (6,7) & 76 \\
\hline Juillet 1997 et janvier $1998(6,7)$ & 100 \\
\hline 1994 à 1996 (2) & 34 (pulsovars 3, 3A) \\
\hline
\end{tabular}

Dans la foulée des éclosions survenues dans les dernières années à Montréal, des efforts avaient été entrepris pour tenter de joindre les différents groupes de la CJUO dans le but de prévenir la transmission des maladies infectieuses et, en particulier, la transmission des maladies entériques dans cette communauté. Ces efforts ont permis d'établir des liens privilégiés avec deux organisations communautaires juives ayant des rapports avec les divers groupes de la CJUO de la région de Montréal. Par leur intermédiaire, des messages préventifs provenant de la DRSP ont pu être acheminés à ces groupes qui ont des contacts limités à l'extérieur de leur population.

Dès que l'éclosion a été suspectée, une notification et une communication ont été transmises aux deux organisations communautaires juives traitant avec la CJUO. La première organisation a accès à une clinique médicale, un lien téléphonique en yiddish avec plus de 2000 familles juives, tout particulièrement les groupes ultra-orthodoxes, ainsi qu'un lien avec les synagogues. La seconde organisation a des conseillers qui travaillent dans le milieu communautaire (milieu de garde, milieu scolaire). Ces deux organisations ont participé à l'effort de la DRSP pour rendre accessible en temps opportun l'information sur l'éclosion de shigellose en cours et sur les moyens à mettre en place pour prévenir et contrôler la transmission de cette maladie. Des affiches sur le lavage des mains en français, en anglais et en yiddish pour les enfants et les adultes ont été distribuées pour sensibiliser les enfants et les travailleurs des milieux de garde et des écoles. Nous supposons que ces communications acheminées précocement aux familles, aux intervenants des milieux de garde et scolaires ainsi qu'aux partenaires communautaires de la CJUO sur les mesures préventives ont pu contribuer à réduire la transmission de la shigellose. Même si l'éclosion semblait persister après le 25 mars, les messages préventifs véhiculés auprès de la CJUO ont pu réduire l'ampleur de l'éclosion.

La diminution du nombre de cas observé sur la courbe épidémique entre le 5 avril et le 3 mai pourrait être liée en partie à la fête de la Pâque Juive qui s'est déroulée du 3 au 11 avril. La fermeture des milieux de garde et scolaire durant cette période a pu réduire la transmission.

Les souches de $S$. sonnei provenant des cas confirmés montrent une résistance à l'ampicilline et au TMP-SMX. La résistance de Shigella aux antimicrobiens de première intention (ampicilline et TMP-SMX) est considérée comme une menace sérieuse par les Centers for Disease Control and Prevention (11). Cette situation amène le clinicien à utiliser davantage d'autres 
antimicrobiens comme la ciprofloxacine ou l'azithromycine alors que des infections résistantes à ces deux antibiotiques sont déjà rapportées.

Le profil électrophorétique de la souche isolée dans la présente investigation (pulsovar 148) a montré que cette souche n'avait pas été rapportée jusqu'à maintenant au Québec et était distincte de celle responsable de l'éclosion newyorkaise. Étant donné que les souches de Shigella ne font pas l'objet d'une surveillance systématique en laboratoire, il ne peut être exclu que cette souche circule depuis un certain temps à Montréal ou dans d'autres régions. La surveillance en laboratoire des souches de Shigella pourrait certainement faciliter la surveillance épidémiologique dans certains groupes à risque.

La présente investigation comporte plusieurs limites. Seuls les cas confirmés par une analyse de laboratoire sont recensés dans le présent rapport. Les renseignements recueillis lors de l'enquête des cas laissent penser que le nombre de cas déclarés représente une sous-estimation du nombre réel de cas. En effet, des cas de diarrhée survenus dans plusieurs familles n'ont pas été confirmés, ni déclarés à la DRSP. Bien que la transmission intrafamiliale et communautaire dans les milieux de garde et scolaire soit fortement soupçonnée, la source d'exposition probable était inconnue pour un bon nombre de cas au moment de l'enquête.

\section{Conclusion}

Cette investigation décrit une éclosion d'infection à S. sonnei dans la CJUO de Montréal qui a touché principalement les enfants d'âge préscolaire et scolaire. La mise en évidence d'une transmission de personne à personne dans une communauté qui a des contacts limités à l'extérieur de leur population souligne l'importance de maintenir et de consolider des liens avec des partenaires de la CJUO de façon à prévenir et à intervenir rapidement en cas d'éclosion. Avec ces partenaires, il est possible de promouvoir et de renforcer auprès des adultes (parents, éducateurs et professeurs) les mesures préventives ayant démontré une efficacité dans la prévention et le contrôle des maladies infectieuses et, en particulier, des éclosions de Shigella (ex. : lavage des mains supervisés chez les enfants, décontamination des jouets ou autres objets partagés, retrait temporaire des enfants avec diarrhée des milieux de garde et scolaires).

\section{Remerciements}

Nous remercions tous les enquêteurs qui ont investigué les cas et qui sont intervenus auprès des familles et des milieux. Nous voulons également remercier les responsables et les intervenants des deux organisations communautaires juives pour leur travail auprès de la CJUO, ainsi que la $D^{\text {re }}$ Sandra Palmieri, le Dr Robert Allard, $\mathrm{M}^{\text {me }}$ Maryse Lapierre et la $\mathrm{D}^{\text {re }}$ Carole Morissette pour leurs commentaires.

\section{Conflit d'intérêts}

Aucun.

\section{Références}

1. New York City Department of Health and Mental Hygiene. 2014 Alert \#39: Outbreak of shigellosis in Borough Park and Williamsburg, December 17, 2014 ; https://a816health29ssl.nyc.gov/sites/NYCHAN/Lists/ AlertUpdateAdvisoryDocuments/HAN_Shigella.pdf.

2. Sobel J, Cameron DN, Ismail J, Strockbine N, Williams M; Diaz PS, et al. A prolonged outbreak of Shigella sonnei infections in traditionally observant Jewish communities in North America caused by a molecularly distinct bacterial subtype. J Infect Dis. 1998 May;177(5):1405-1409.

3. Garrett V, Bornschlegel K, Lange D, Reddy V, Kornstein L, Kornblum J, et al. A recurring outbreak of Shigella sonnei among traditionally observant Jewish children in New York City: the risks of daycare and household transmission. Epidemiol Infect. 2006;134(6):1231-6.

4. Daudens E, Dejour-Salamanca D, Isnard H, Mariani-Kurkdjian P, Filliol I, Bingen E. Épidémie de gastro-entérites aiguës à Shigella sonnei résistantes à l'amoxicilline, au cotrimoxazole et à l'azithromycine en Île-de-France - Janvier - Avril 2007. Saint-Maurice (Fra) : Institut de veille sanitaire, décembre 2009. 18 p. www.invs.sante.fr.

5. De Schrijver K, Bertrand S, Gutierrez Garitano I, Van den Branden D, Van Schaeren J. Outbreak of Shigella sonnei infections in the Orthodox Jewish community of Antwerp, Belgium, April to August 2008. Euro Surveill. 2011;16(14):pii=19838.

6. Andermann A. Shigellosis in the Montreal Jewish community: review of cases and recommended control measures. Direction de santé publique, Région de Montréal; 2005. $35 \mathrm{p}$.

7. Hannah H. Bilan des éclosions d'infections à Shigella sonnei, Montréal et au Québec, 5 août 2007 au 26 janvier 2008. Direction de santé publique, Région de Montréal; 2008. $21 \mathrm{p}$.

8. Gardhouse C. Shigellose dans la communauté juive orthodoxe, Montréal, août 2011 - décembre 2012. Direction de santé publique, Région de Montréal; 2013. 31 p.

9. Susser S. Recurrent shigellosis outbreaks in the Montreal Jewish community: case study in health inequality. Direction de santé publique, Région de Montréal; 2012. 35 p.

10. Centers for Disease Control and Prevention (CDC). Antibiotic resistance threats in the United States, 2013;113 p. http://www.cdc.gov/drugresistance/pdf/arthreats-2013-508.pdf.

11. Calderon-Margalit R, Navon-Venezia S, Gefen D et al. Biennial hyperepidemic shigellosis in an observant Jewish community. Epidemiol Infect. 2010;138:244-252. 